\title{
Switching based limit cycle control for compliantly actuated second-order systems
}

\author{
Dominic Lakatos* Alin Albu-Schäffer* \\ * German Aerospace Center (DLR), Institute of Robotics and \\ Mechatronics, D-82234 Oberpfaffenhofen, Germany; e-mail: \\ dominic.lakatos@dlr.de
}

\begin{abstract}
This paper derives a stability statement for a novel, switching based limit cycle control. The stability proof is based on multiple Lyapunov functions and a new interpretation of contraction analysis. By showing that the dissipated energy on the cycle increases with increasing velocity, while the injected energy is constant, the emergence of an attractive limit cycle is shown. The approach applies for general, nonlinear, and compliantly actuated secondorder systems, with positive definite plant parameters and non-aperiodic solutions. An analysis of the controller parameters reveals, that for the majority of parameters, global attractiveness of the limit cycle can be guaranteed.
\end{abstract}

\section{INTRODUCTION}

In the field of robot control, mechanical systems including nonlinear compliances have recently gained increasing attention. The elasticity in the actuation can be exploited to robustly handle impacts and unknown contact forces at one hand, and is able to store and targetedly release potential energy at the other hand (Grebenstein and Smagt (2008); Albu-Schäffer et al. (2011); Braun et al. (2011) Haddadin et al. (2012)). Especially, the capability to store potential energy can be exploited in periodic motions tasks such as hammering, and drumming, or jumping, walking, and running. Thereby, the energy efficiency of the motion can be increased and the required peak power of the actuators can be reduced.

On the basis of the seminal work of Van der Pol (1926), several control methods to implement a limit cycle behavior in robotic systems have been proposed by Stramigioli and Dijk (2008); Garofalo et al. (2013); Lakatos et al. (2013a). These controllers change the original dynamics of the plant by a nonlinear damping term that increases the system energy along trajectories inside the limit cycle and decreases the system energy outside the limit cycle. Thereby, the original dynamics of the plant is substantially changed. In particular, applying this approach to robotic systems entails additional energy losses in the actuators even in phases of positive damping, since the nonlinear damping is generated artificially by control.

In our previous work Lakatos et al. (2013c), we introduced a substantially different control concept to excite periodic motions in compliantly actuated mechanical systems. The controller switches the equilibrium position of the elastic elements triggered by the force acting in the springs. Thereby, energy is induced in the system. The concept is based on the assumption that the plant has intrinsic damping properties such that the energy induced by the switchings is dissipated in the phases between the switching. This results in a cyclic motion where the energy exchange over one cycle is balanced. By showing that the dissipated en- ergy on the cycle increases with increasing velocity, while the injected energy is constant, the emergence of a stable and attractive limit cycle will be deduced. In our recent works Lakatos et al. (2013b, 2014), we applied ${ }^{1}$ this novel $^{-}$ concept to control multi-degree-of-freedom cyclic motions and practically verified the robustness and efficiency of the method for complex-structured systems including ground contacts as appearing, for instance, in legged robotic systems. In particular, in Lakatos et al. (2013b), we combine the switching based limit cycle control with the notion of intrinsic mechanical oscillations modes, which further supports the idea of exploiting the mechanical properties of the plant. Note that the above mentioned control methods are implemented for $2 n$ th-order (robotic) systems which either display dominant second-order dynamics (Lakatos et al. $(2013 \mathrm{c})$ ) or can be reduced by control to a secondorder system (Lakatos et al. (2013a)).

This work derives a comprehensive stability statement for the switching based limit cycle control of a nonlinear, compliantly actuated second-order system. Stability of the hybrid system is deduced based on multiple, physically motivated Lyapunov functions (Branicky (1998)) and a novel interpretation of contraction analysis, which differs from what was proposed by Lohmiller and Slotine (1998). Furthermore, the design of the controller parameters is analysed. Both, the stability analysis and the controller design exemplify the basic principle of this alternative concept generating limit cycles.

The paper is organized as follows: Section 2 introduces the controller and investigates the design of the controller parameters. Section 3 treats the stability analysis. Thereby, Section 3.2 introduces the main theorem and the preceding subsections validate the assumptions of this theorem for the control system discussed in Section 2. In particular, Section 3.5 presents a second theorem to prove the basic assumption of the main theorem. This theorem represents a new interpretation of contraction analysis and therefore

\footnotetext{
1 Videos showing applications of the switching based limit cycle control can be found at www.robotic.dlr.de/index.php?id=357 .
} 


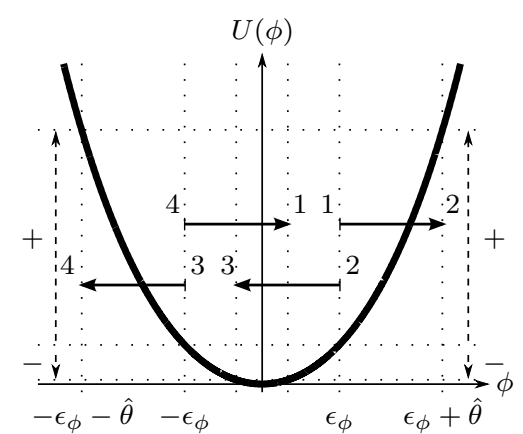

(a) $\epsilon_{\phi}>0,|\hat{\theta}|<2 \epsilon_{\phi}$

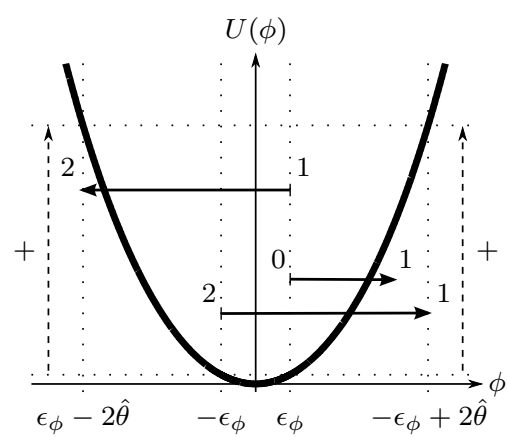

(b) $\epsilon_{\phi}>0,|\hat{\theta}| \geq 2 \epsilon_{\phi}$

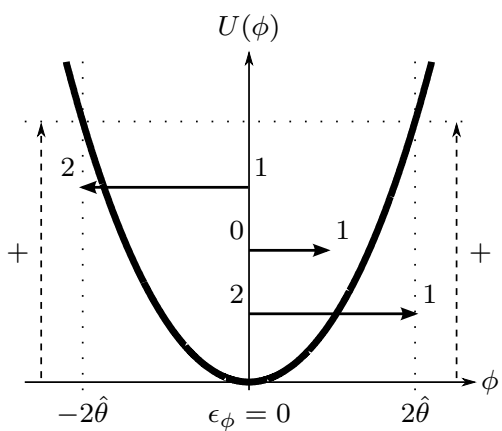

(c) $\epsilon_{\phi}=0,|\hat{\theta}|>0$

Fig. 1. Switching behavior of the potential energy. Three different sets of controller parameters $\left(\epsilon_{\phi}, \hat{\theta}\right)$ are compared. The vertical, dashed arrows represent the change of potential energy due to the switching and the " +" /" - signs indicate the energy input/output. The horizontal, solid arrows represent the switching of the deflection $\phi$. The numbers at the start and end points of the arrows represent the transition of the switching signal $\sigma$.

may be seen as a separate result of the paper. Concluding remarks are given in Section 4. Finally, the theorems introduced in Section 3 are proved in Appendix A and B.

\section{CONTROLLER DESIGN}

Consider the system

$$
m \ddot{q}+d \dot{q}=-\frac{\partial U(\phi)}{\partial \phi},
$$

where $\phi=q-\theta$ is the deflection of a mechanical spring, $\theta$ is the control input, $\boldsymbol{x}=(q, \dot{q})^{T} \in \mathbb{R}^{2}$ are continuous states, $d, m>0$ are damping and (generalized) inertia constants, respectively. The (elastic) potential $U: \mathbb{R} \rightarrow \mathbb{R}_{\geq 0}$ is positive definite and the (generalized) force

$$
\tau(\phi):=-\partial U(\phi) / \partial \phi
$$

is an odd and monotone function of the deflection $\phi$. Then consider the switching control introduced in Lakatos et al. (2013c)

$$
\theta\left(q, \theta_{-}\right)= \begin{cases}-\operatorname{sign}\left(\phi\left(q, \theta_{-}\right)\right) \hat{\theta} & \text { if }\left|\phi\left(q, \theta_{-}\right)\right|>\epsilon_{\phi} \\ 0 & \text { otherwise }\end{cases}
$$

where $\epsilon_{\phi} \geq 0$ is a constant threshold, $\hat{\theta}>0$ a switching amplitude, and $\theta_{-}$the state of $\theta$ before the switching. The discontinuous control (3) switches the equilibrium position $\theta$ of the spring (2) triggered by the the deflection $\phi$.

Due to the switching nature of the control law, the closedloop system (1) and (3) can be regarded as a (continuoustime) autonomous hybrid system (Branicky, 1998, Sect. I-B):

$$
\begin{aligned}
& \dot{\boldsymbol{x}}(t)=\boldsymbol{f}(\boldsymbol{x}(t), \theta(t))=\frac{1}{m}\left(\begin{array}{c}
\dot{q}(t) \\
\tau(q(t)-\theta(t))-d \dot{q}(t)
\end{array}\right) \\
& \theta(t)=\theta\left(q(t), \theta_{-}\right)=\left\{\begin{aligned}
\hat{\theta} & \text { if } q(t)-\theta_{-}<-\epsilon_{\phi} \\
0 & \text { if }-\epsilon_{\phi} \leq q(t)-\theta_{-} \leq \epsilon_{\phi} \\
-\hat{\theta} & \text { if } q(t)-\theta_{-}>\epsilon_{\phi}
\end{aligned}\right.
\end{aligned}
$$

where $\boldsymbol{x}(t)=(q, \dot{q})^{T} \in \mathbb{R}^{2}$ and $\theta(t) \in \mathcal{T}:=\{-\hat{\theta}, 0, \hat{\theta}\}$. The mapping $\boldsymbol{f}(\cdot, \theta): \mathbb{R}^{2} \rightarrow \mathbb{R}^{2}$ is globally Lipschitz continuous for each $\theta \in \mathcal{T}$, and $\theta: \mathbb{R} \times \mathcal{T} \rightarrow \mathcal{T}$ is finite. Therefore, (4) represents the continuous dynamics and (5) represents the finite dynamics ${ }^{2}$.

2 Note that $\theta_{-}$indicates that the finite state is piecewise continuous from the right.
Remark 1. An intuitive interpretation of the hybrid system (4) and (5) is that the finite dynamics (5) switches the potential of the continuous dynamics (4) at a fixed level $U\left(\epsilon_{\phi}\right)$ by constant amounts $\Delta U_{ \pm}=U\left(\epsilon_{\phi} \pm \hat{\theta}\right)-U\left(\epsilon_{\phi}\right)$.

In the following we consider a simplified representation of the hybrid system which is equivalent to (4) and (5) and can be written in the form

$$
\dot{\boldsymbol{x}}_{\sigma}=\boldsymbol{f}_{\sigma}\left(\boldsymbol{x}_{\sigma}\right) \text {. }
$$

Here, each state of the switching signal $\sigma(q(t), \theta(t)) \in$ $\Sigma:=\{1,2, \ldots, n\}$ corresponds to one of the piecewise continuous vector fields $\boldsymbol{f}_{\sigma}(\cdot): \mathbb{R}^{2} \rightarrow \mathbb{R}^{2}$. Therefore, a number of $n$ subsystem with corresponding hybrid states $\boldsymbol{x}_{\sigma}=\left(\phi_{\sigma}, \dot{q}\right)^{T} \in \mathbb{R}^{2}$ result, where $\phi_{\sigma}$ is defined such that $\frac{\mathrm{d}}{\mathrm{d} t} \phi_{\sigma}=\dot{q}$. Note that there exists a one-to-one correspondence

$$
\boldsymbol{x}=\chi_{\sigma}\left(\boldsymbol{x}_{\sigma}\right),
$$

between the hybrid states $\boldsymbol{x}_{\sigma}$ and continuous states $\boldsymbol{x}$ for each $\sigma \in \Sigma$ fixed.

In the following subsections, the reduced representation (6) of the hybrid system (4) and (5) will be explained in detail using the visual tool of directed graphs as introduced by Tavernini (1987). Thereby, we will distinguish three different sets of controller parameters $\left(\epsilon_{\phi}, \hat{\theta}\right)$, which lead to different switching behaviors as illustrated in Fig. 1.

2.1 Hybrid system for $\epsilon_{\phi}>0,|\hat{\theta}|<2 \epsilon_{\phi}$

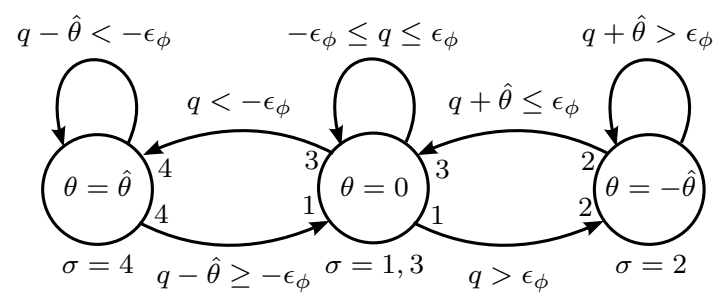

Fig. 2. Directed graph representing the finite dynamics for controller parameters $\left(\epsilon_{\phi}, \hat{\theta}\right) \in \mathcal{P}_{1}$. Each vertex represent a state of the finite dynamics / switching signal. Each arrow represents a transition. 
In case $\left(\epsilon_{\phi}, \hat{\theta}\right) \in \mathcal{P}_{1}$, where

$$
\mathcal{P}_{1}:=\left\{\left(\epsilon_{\phi}, \hat{\theta}\right) \in \mathbb{R}^{2}\left|\epsilon_{\phi}>0,\right| \hat{\theta} \mid<2 \epsilon_{\phi}\right\},
$$

the vector field in (6) takes the form

$$
\boldsymbol{f}_{\sigma}\left(\boldsymbol{x}_{\sigma}\right)=\left(\begin{array}{ll}
\frac{1}{\dot{q}} & \text { if } \sigma=1 \text { or } 3 \\
\left.\frac{1}{m}(q)-d \dot{q}\right)-d \dot{q} & \text { if } \sigma=2 \\
\tau(q-\hat{\theta})-d \dot{q} & \text { if } \sigma=4
\end{array}\right) .
$$

The finite dynamics that selects the specific continuous dynamics in (8) (dependent on the state of the switching signal $\sigma$ ) is illustrated in Fig. 2. Thereby, the corresponding ordered switching sequence $\Sigma_{\mathcal{P}_{1}}=\{1,2,3,4\}$ occurs and is repeated, if the appropriate transition condition is satisfied. Note that the switching sequence can be initialized for any initial state $\sigma(0) \in \Sigma_{\mathcal{P}_{1}}$. Moreover, the mapping (7) takes the form

$$
\chi_{\sigma}=\left(\begin{array}{ll}
q & \text { if } \sigma=1 \text { or } 3 \\
q+\hat{\theta} & \text { if } \sigma=2 \\
q-\hat{\theta} & \text { if } \sigma=4 \\
\dot{q}
\end{array}\right)
$$

2.2 Hybrid system for $\epsilon_{\phi}>0,|\hat{\theta}| \geq 2 \epsilon_{\phi}$

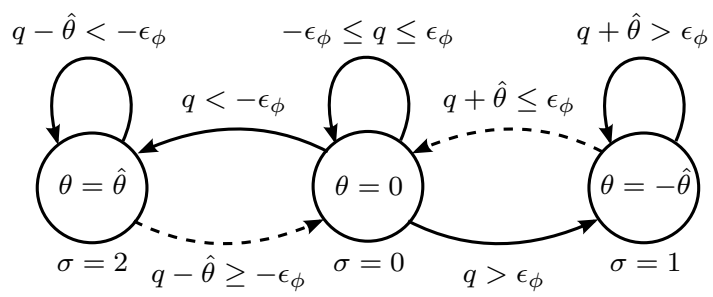

Fig. 3. Directed graph representing the finite dynamics for controller parameters $\left(\epsilon_{\phi}, \hat{\theta}\right) \in \mathcal{P}_{2} \cup \mathcal{P}_{3}$. Each vertex represent a state of the finite dynamics / switching signal. Each arrow represents a transition. Dashed arrows represent transitions that instantaneously evolve to the next transition in the same direction, 1.e. only the state $\sigma=0$ can be stable initially (choosing initial conditions $\theta(0)=0$ and $\left.-\epsilon_{\phi} \leq q(0) \leq \epsilon_{\phi}\right)$.

In case $\left(\epsilon_{\phi}, \hat{\theta}\right) \in \mathcal{P}_{2}$, where

$$
\mathcal{P}_{2}:=\left\{\left(\epsilon_{\phi}, \hat{\theta}\right) \in \mathbb{R}^{2}\left|\epsilon_{\phi}>0,\right| \hat{\theta} \mid \geq 2 \epsilon_{\phi}\right\},
$$

the vector field in (6) takes the form

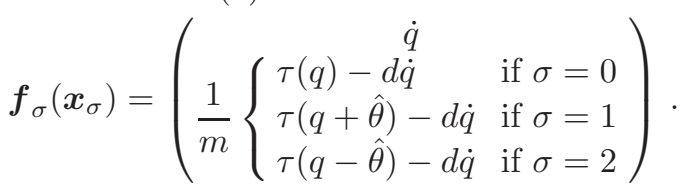

The finite dynamics (Fig. 3) that selects the specific continuous dynamics in (11) has two stable states (1.e. $\sigma=1$ and $\sigma=2$ ) and one only initially stable state (1.e. $\sigma=0)$. This is as the transition from $\sigma=1 \rightarrow 0$ already satisfies the condition for a transition from $\sigma=0 \rightarrow 2$ and vice versa. Therefore, the fundamental ordered switching sequence $\Sigma_{\mathcal{P}_{2}}=\{1,2\}$ is repeated for all initial states $\sigma(0) \in \Sigma_{\mathcal{P}_{2}}$. Moreover, the mapping (7) takes the form

$$
\chi_{\sigma}=\left(\begin{array}{ll}
q & \text { if } \sigma=0 \\
q+\hat{\theta} & \text { if } \sigma=2 \\
q-\hat{\theta} & \text { if } \sigma=4 \\
\dot{q}
\end{array}\right) .
$$

\subsection{Hybrid system for $\epsilon_{\phi}=0,|\hat{\theta}|>0$}

The case $\left(\epsilon_{\phi}, \hat{\theta}\right) \in \mathcal{P}_{3}$, where

$$
\mathcal{P}_{3}:=\left\{\left(\epsilon_{\phi}, \hat{\theta}\right) \in \mathbb{R}^{2}\left|\epsilon_{\phi}=0,\right| \hat{\theta} \mid>0\right\},
$$

is a special case of the hybrid system introduced in Section 2.2, equipped with the equivalent ordered switching sequence, 1.e. $\Sigma_{\mathcal{P}_{3}} \equiv \Sigma_{\mathcal{P}_{2}}$. The only difference is that even the initial conditions $\boldsymbol{x}_{\sigma=0}(0)=\mathbf{0}$ are unstable.

\section{STABILITY ANALYSIS}

\subsection{Notation}

Throughout we require to refer to subsystems resulting from (6), (8), and (11). Therefore, the index $j$ denotes the subsystem $\dot{\boldsymbol{x}}_{j}=\boldsymbol{f}_{j}\left(\boldsymbol{x}_{j}\right)$, where $j=\sigma \in \Sigma_{\mathcal{P}_{1}} \cup \Sigma_{\mathcal{P}_{2}} \cup \Sigma_{\mathcal{P}_{3}}$. Further, we require to specify the time instants of the switching. Therefore, we introduce the subscripts $(\cdot)_{j^{ \pm}}$, where $j$ denotes the subsystem, $k$ counts the iteration, and the signs "+" and "-" denote the time instant when the subsystem is "switched on" respectively "switched off". For instance, $t_{j_{k^{+}}}<t_{j_{k^{-}}}$denote the $k$-th time instants where the $j$-th subsystem is "switched on" and "switched off", respectively. For functions of time and states, we use the same notation. That is $V_{j_{k+}}:=V\left(t_{j_{k+}}\right)$ and $\boldsymbol{x}_{j_{k+}}:=\boldsymbol{x}\left(t_{j_{k+}}\right)$.

Some additional generic notations are introduced in advance:

- energy exchange in the active region of subsystem $j$ : $\Delta V_{j_{k}}:=V_{j}\left(\boldsymbol{x}_{j_{k^{-}}}\right)-V_{j}\left(\boldsymbol{x}_{j_{k+}}\right)$;

- energy exchange due to the switching from subsystem $j$ to $j+1: \Delta U_{j, j+1} \equiv \Delta V_{j, j+1}:=V_{j+1}\left(\boldsymbol{x}_{(j+1)_{k+}}\right)-$ $V_{j}\left(\boldsymbol{x}_{j_{k-}}\right)=$ const.;

- the index $(j+1) \in \Sigma_{\mathcal{P}_{i}}$ has the value $\min \left(\Sigma_{\mathcal{P}_{i}}\right)=1$ if $j=\max \left(\Sigma_{\mathcal{P}_{i}}\right)$;

- the energy exchange of the repetition $k$ at $l \in \Sigma_{\mathcal{P}_{i}}$ is denoted

$$
\Delta V_{\left(\Sigma_{\mathcal{P}_{i}}\right)_{k}}^{l}=\sum_{j=l}^{l+(n-1)}\left(\Delta V_{j_{k}}+\Delta U_{j, j+1}\right),
$$

in particular, $\Delta V_{\left(\Sigma_{\mathcal{P}_{i}}\right)_{k}}^{l} \equiv \Delta V_{l_{(k+1)^{+}}}$.

\subsection{Stability statement}

The idea of the stability statement is based on multiple Lyapunov functions for the hybrid system as in Branicky (1998); Lu and Brown (2010); Zhao and Hill (2005, 2008). Therefore we define scalar function $V_{j}: \mathbb{R}^{2} \rightarrow \mathbb{R}_{>0}$, representing the physical energy of the subsystem $j$. The functions are chosen such that they are non-increasing in the "active" time interval $\left[t_{k^{+}}, t_{j_{k^{-}}}\right]$. Then we observe the progress of one of the energy functions at e.g. the "switch on" time instant $V_{j_{k^{+}}}$. If the difference of this value between the $(k+1)$-th and $k$-th repetition converges to zero, the trajectory reaches a steady state, where a certain point in the state space is reached repeatedly. Notice that our stability statement differs from what was done in the above mentioned papers, since it adds a condition for the existence of limit cycles. This motivates the following theorem: 
Theorem 2. Consider the autonomous hybrid second order system defined by (6), where the fundamental switching sequence $\Sigma$ is repeated. Suppose we have Lyapunov functions $V_{j}(t)$ for each individual subsystem, non-increasing for all $t \in \bigcup_{k}\left[t_{j_{k+}}, t_{j_{k^{-}}}\right], j \in \Sigma, k \in \mathbb{N}$. If for all $j \in \Sigma$ and $k \in \mathbb{N}$

$$
\Delta V_{j_{(k+1)+}}:=V_{j_{(k+1)^{+}}}-V_{j_{k^{+}}}<c
$$

keeps bounded from above and one of the two following conditions is satisfied,

(C1) the switching sequence $\Sigma$ is finite such that the hybrid system stays in one subsystem,

(C2) for at least one $j$, the sequence $\left\{\Delta V_{j_{(k+1)}}\right\}$ converges to zero, as $k \rightarrow \infty$,

the solution of the hybrid system keeps bounded. In case condition (C2) is satisfied and if additionally

(C3) the equilibrium points $\boldsymbol{x}_{j}=\mathbf{0}$ of the individual subsystems $\dot{\boldsymbol{x}}_{j}=\boldsymbol{f}_{j}\left(\boldsymbol{x}_{j}\right)$ are globally asymptotically stable,

then the continuous state trajectory $\boldsymbol{x}(t)$ approaches a limit cycle.

Proof. The proof of the above theorem is given in the Appendix A.

In the remainder of this section we will show that the closed-loop system (1) and (3) satisfies the conditions of Theorem 2.

\subsection{Lyapunov functions}

Consider Lyapunov function candidates

$$
V_{j}\left(\boldsymbol{x}_{j}\right)=T(\dot{q})+U\left(\phi_{j}\right)
$$

for each individual subsystem resulting from (6) together with (8) and (11), respectively. Each $V_{j}\left(\boldsymbol{x}_{j}\right)$ in (13) represents the sum of the kinetic energy $T(\dot{q})=\frac{1}{2} m \dot{q}^{2}$ and potential energy $U\left(\phi_{j}\right)$ of the plant (1). Due to the definition of $U$ (cf. (2)), $V_{j}(\mathbf{0})=0$ and the derivative along the solution of the corresponding subsystem $\dot{V}_{j}\left(\boldsymbol{x}_{j}\right)=-d \dot{q}^{2} \leq 0$ is non-positive $\forall \boldsymbol{x}_{j} \in \mathbb{R}^{2}$. Therefore, $V_{j}\left(\boldsymbol{x}_{j}\right)$ are Lyapunov functions for each individual subsystem $j$. Moreover, since $\left\{\boldsymbol{x}_{j} \in \mathbb{R}^{2} \mid \phi_{j} \neq 0, \dot{q}=0\right\}$ is not an invariant set, the equilibrium point $\boldsymbol{x}_{j}=\mathbf{0}$ of the individual subsystem $j$ is globally asymptotically stable (La Salle's Theorem, see e.g. (Slotine and Li, 1991, Theorem 3.4)). Therefore, the subsystems resulting from (6), (8), and (11) satisfy condition (C3) of Theorem 2.

\subsection{Boundedness of the energy exchange $\Delta V_{\left(\Sigma_{\mathcal{P}_{i}}\right)_{k}}^{l}$}

In the following we check the boundedness conditions of Theorem 2 for different parameter sets $\mathcal{P}_{1}, \mathcal{P}_{2}$, and $\mathcal{P}_{3}$, separately.

In case $\left(\epsilon_{\phi}, \hat{\theta}\right) \in \mathcal{P}_{1}$, the energy exchange of the repetition $k$ at $j=1$ comprises

$$
\begin{aligned}
\Delta V_{\left(\Sigma_{\mathcal{P}_{1}}\right)_{k}}^{1} & =\Delta V_{1_{k}}+\Delta U_{1,2}+\Delta V_{2_{k}}+\Delta U_{2,3} \\
& +\Delta V_{3_{k}}+\Delta U_{3,4}+\Delta V_{4_{k}}+\Delta U_{4,1} .
\end{aligned}
$$

Considering the switching behavior discussed in Section 2.1 (which is illustrated in Fig. 1(a)) and since the equilibrium point $\boldsymbol{x}_{j}=\mathbf{0}$ of each individual subsystem $\dot{\boldsymbol{x}}_{j}=\boldsymbol{f}_{j}\left(\boldsymbol{x}_{j}\right)$ is globally asymptotically stable (Section 3.3$)$, it follows that

$$
\begin{aligned}
\Delta U_{1,2} & =\Delta U_{3,4}=c_{1}:=U\left(\epsilon_{\phi}+\hat{\theta}\right)-U\left(\epsilon_{\phi}\right)>0 \\
-\Delta U_{2,3} & =-\Delta U_{4,1}=c_{2}:=U\left(\epsilon_{\phi}\right)-U\left(\epsilon_{\phi}-\hat{\theta}\right)>0 \\
\Delta V_{1_{k}} & =\Delta T_{1_{k}}+c_{2}<0 \Longrightarrow \Delta T_{1_{k}}<-c_{2} \\
\Delta V_{2_{k}} & =\Delta T_{2_{k}}-c_{1}<0 \Longrightarrow \Delta T_{2_{k}}<c_{1} \\
\Delta V_{3_{k}} & =\Delta T_{3_{k}}+c_{2}<0 \Longrightarrow \Delta T_{3_{k}}<-c_{2} \\
\Delta V_{4_{k}} & =\Delta T_{4_{k}}-c_{1}<0 \Longrightarrow \Delta T_{4_{k}}<c_{1}
\end{aligned}
$$

where $c_{1}>c_{2}$ are positive constants. Accordingly, (14) can be compactly written

$$
\Delta V_{\left(\Sigma_{\mathcal{P}_{1}}\right)_{k}}^{1}=\sum_{j=1}^{4} \Delta T_{j_{k}}<c=2\left(c_{1}-c_{2}\right)>0,
$$

where it can be seen that the boundedness condition of Theorem 2 is satisfied.

In case $\left(\epsilon_{\phi}, \hat{\theta}\right) \in \mathcal{P}_{2}$, the energy exchange of the repetition $k$ at $j=1$ comprises

$$
\Delta V_{\left(\Sigma_{\mathcal{P}_{2}}\right)_{k}}^{1}=\Delta V_{1_{k}}+\Delta U_{1,2}+\Delta V_{2_{k}}+\Delta U_{2,1} .
$$

Thereby, it is assumed that the system is in a state, where it initially reached subsystem 1 . Considering the switching behavior discussed in Section 2.2 (which is illustrated in Fig. 1(b)) and since the equilibrium point $\boldsymbol{x}_{j}=\mathbf{0}$ of each individual subsystem $\dot{\boldsymbol{x}}_{j}=\boldsymbol{f}_{j}\left(\boldsymbol{x}_{j}\right)$ is globally asymptotically stable (Section 3.3), it follows that

$$
\begin{aligned}
\Delta U_{1,2} & =\Delta U_{2,1}=c_{1}:=U\left(-\epsilon_{\phi}+2 \hat{\theta}\right)-U\left(\epsilon_{\phi}\right)>0 \\
\Delta V_{1_{k}} & =\Delta T_{1_{k}}-c_{1}<0 \Longrightarrow \Delta T_{1_{k}}<c_{1} \\
\Delta V_{2_{k}} & =\Delta T_{2_{k}}-c_{1}<0 \Longrightarrow \Delta T_{2_{k}}<c_{1}
\end{aligned}
$$

where $c_{1}$ is a positive constant. Then, (16) reduces to

$$
\Delta V_{\left(\Sigma_{\mathcal{P}_{2}}\right)_{k}}^{1}=\sum_{j=1}^{2} \Delta T_{j_{k}}<c=2 c_{1}>0
$$

where it can be seen that the boundedness condition of Theorem 2 is satisfied.

The case $\left(\epsilon_{\phi}, \hat{\theta}\right) \in \mathcal{P}_{3}$ is similar to the case $\left(\epsilon_{\phi}, \hat{\theta}\right) \in \mathcal{P}_{2}$, except that the repeated switching sequence $\Sigma_{\mathcal{P}_{3}}$ is reached for all initial conditions $\boldsymbol{x}(0) \neq \mathbf{0}$. The energy exchange of the repetition $k$ at $j=1$ has the same form as (16). The corresponding properties

$$
\begin{aligned}
\Delta U_{1,2} & =\Delta U_{2,1}=c_{3}:=U(2 \hat{\theta})>0 \\
\Delta V_{1_{k}} & =\Delta T_{1_{k}}-c_{3}<0 \Longrightarrow \Delta T_{1_{k}}<c_{3} \\
\Delta V_{2_{k}} & =\Delta T_{2_{k}}-c_{3}<0 \Longrightarrow \Delta T_{2_{k}}<c_{3}
\end{aligned}
$$

where $c_{3}$ is a positive constant, can be derived from Fig. 1(c) such that

$$
\Delta V_{\left(\Sigma_{\mathcal{P}_{3}}\right)_{k}}^{1}=\sum_{j=1}^{2} \Delta T_{j_{k}}<c=2 c_{3}>0 .
$$

It can be seen that the boundedness condition of Theorem 2 is satisfied.

\subsection{Convergence of the sequence $\left\{\Delta V_{\left(\Sigma_{\mathcal{P}_{i}}\right)_{k}}^{l}\right\}$ to zero}

The convergence of the sequence

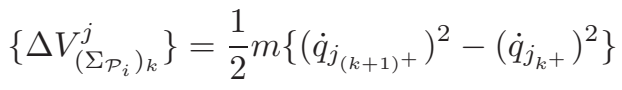




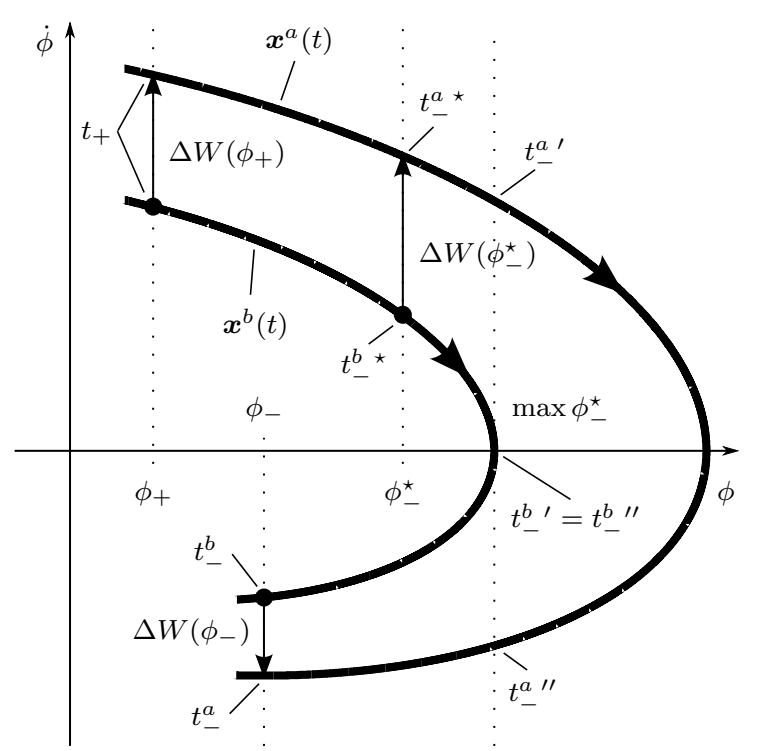

Fig. 4. Contraction behavior of displacements measured at fixed position. The specified time instants are used in the proof in Appendix B.

to zero will be shown using a concept inspired by contraction analysis for nonlinear systems, introduced in Lohmiller and Slotine (1998). The contraction of nominal and "actual" trajectories corresponding to $\Delta V_{\left(\Sigma_{\mathcal{P}_{i}}\right)_{\infty}}^{j}=$ 0 and $\Delta V_{\left(\Sigma_{\mathcal{P}_{i}}\right)_{k}}^{j} \neq 0$, respectively, will lead us to the conclusion that the sequence has converged. Instead of using the notion of virtual displacements, which describe the displacement of two neighboring trajectories at fixed time, we measure the distance between two trajectories at fixed position. This is required since the considered hybrid system switches position dependently and therefore neighboring trajectories of each individual subsystem $j$ are starting and ending always at constant positions $\phi_{j_{k}+}$ and $\phi_{j_{k-}}$, respectively. This in turn leads to different time durations of the neighboring trajectories for the general, nonlinear case of the system (1). For this reason, we introduce a different notion of displacements which describes the distance of neighboring trajectories (measured with respect to a metric) when the position is held constant.

Let us now derive the idea of the convergence principle. Consider therefore segments of neighboring trajectories corresponding to the same asymptotically stable system (denoted by $\boldsymbol{x}^{a}(t)$ and $\boldsymbol{x}^{b}(t)$ ), which are delimited at positions $\phi_{+}$and $\phi_{-}$in the state plane (Fig. 4). Assume that both trajectory segments pass through the same quadrants of the state plane such that we can identify sub-segments delimited at positions $\phi_{+}$and $\phi_{-}^{\star}$, where the velocity of the outer trajectory is larger than the velocity of the inner trajectory, 1.e. $\left|\dot{\phi}^{a}\right|>\left|\dot{\phi}^{b}\right|$. Then, considering the physical energy $\boldsymbol{W}(\boldsymbol{x})=\frac{1}{2} m \dot{\phi}+U(\phi)$ of the system as metric to measure the distance of a point on the trajectory with respect to the origin, we will come to the conclusion that the energy loss along the outer trajectory segments is larger than the energy loss at the inner trajectory segment. Therefore, the distance $\Delta W^{a b}$ between $\boldsymbol{x}^{a}$ and $\boldsymbol{x}^{b}$ at the starting position $\phi_{+}$is larger than the distance at the terminal position $\phi_{-}^{\star}$, 1.e. $\Delta W^{a b}\left(\phi_{+}\right)>\Delta W^{a b}\left(\phi_{-}^{\star}\right)$. This principle can be extended for the case where the trajectory segments pass through more than one quadrant of the state plane. Thereby, the energy loss along the outer trajectory will be always larger than the energy loss along the inner trajectory. This motivates the following theorem which makes use of the foregoing definition.

Definition 3. Two trajectories $\boldsymbol{x}^{a}(t)$ and $\boldsymbol{x}^{b}(t)$ of the same second order system $\dot{\boldsymbol{x}}=\boldsymbol{f}(\boldsymbol{x})$, with states $\boldsymbol{x}=(\phi, \dot{\phi})^{T}$, are defined as neighboring trajectories, if they start and terminate at the same positions $\phi_{+}$and $\phi_{-}$, respectively.

Theorem 4. Consider two neighboring trajectories $\boldsymbol{x}^{a}(t)$ and $\boldsymbol{x}^{b}(t)$ of the asymptotically stable system $\dot{\boldsymbol{x}}=\boldsymbol{f}(\boldsymbol{x})$, with states $\boldsymbol{x}=(\phi, \dot{\phi})^{T}$ and a natural metric $W(\boldsymbol{x})=$ $\frac{1}{2} m \dot{\phi}+U(\phi)$ such that $\dot{W}(\boldsymbol{x})=-d \dot{\phi}^{2}$. Suppose there exist trajectory segments defined by:

- $\boldsymbol{x}_{+}^{a}=\left(\phi_{+}, \dot{\phi}_{+}^{a}\right)^{T}, \boldsymbol{x}_{+}^{b}=\left(\phi_{+}, \dot{\phi}_{+}^{b}\right)^{T}$ (starting points), where $\phi_{+}:=\phi^{a}\left(t_{+}\right)=\phi^{b}\left(t_{+}\right)$;

- $\boldsymbol{x}_{-}^{a}=\left(\phi_{-}, \dot{\phi}_{-}^{a}\right)^{T}, \boldsymbol{x}_{-}^{b}=\left(\phi_{-}, \dot{\phi}_{-}^{b}\right)^{T}$ (terminal points), where $\phi_{-}:=\phi^{a}\left(t_{-}^{a}\right)=\phi^{b}\left(t_{-}^{b}\right)$;

- $\boldsymbol{x}_{-}^{a \star}=\left(\phi_{-}^{\star}, \dot{\phi}_{-}^{a \star}\right)^{T}, \boldsymbol{x}_{-}^{b \star}=\left(\phi_{-}^{\star}, \dot{\phi}_{-}^{b}\right)^{T}$ (intermediate points), where $\phi_{-}^{\star}:=\phi^{a}\left(t_{-}^{a \star}\right)=\phi^{b}\left(t_{-}^{b \star}\right)$ such that ${ }^{3}$ $\left|\dot{\phi}^{a}(t)\right|>\left|\dot{\phi}^{b}(t)\right| \geq 0, \forall t \in\left[t_{+} ; \min \left(t_{-}^{a \star}, t_{-}^{b \star}\right)\right] ;$

Then, if both of these trajectory segments pass through the same quadrants of the state plane, the distance $\Delta W^{a b}(\phi)=\left|W^{a}(\phi)-W^{b}(\phi)\right|$ measured at fixed position ${ }^{4}$ is contracting as $\phi$ evolves from $\phi_{+}$to $\phi_{-}$.

Remark 5. Note that in the general nonlinear case, the fact that the velocity on the outer trajectory is larger than on the inner trajectory, does not trivially imply that the dissipated energy is also larger along the outer trajectory, since on the outer trajectory, the cycle period might be shorter.

Proof. The proof of the above theorem is given in the Appendix B.

From Theorem 4 we can conclude that the distance between the nominal limit cycle trajectory and a neighboring trajectory of the individual subsystem $j$ defined by (8) or (11) shrinks as the motions evolves from the "switch on" to the "switch off" position. Since each individual subsystem $j$ of the hybrid system exhibits such a contraction behavior, the distance, for instance, at the "switch on" instant shrink from repetition $k$ to $k+1$. Therefore, condition (C2) of Theorem 2 is satisfied.

\subsection{Comments on the robustness and efficiency of the limit cycle}

In the preceding stability analysis it has been assumed that the transition from subsystem $j$ to subsystem $j+1$ always occurs. This cannot be generally guaranteed. For the switching system (1) with switching control (3) we can distinguish two scenarios which interrupt the switching sequence:

\footnotetext{
3 The trajectory segments connecting the starting points and intermediate points are both in the same quadrant of the state plane.

4 Note that $W^{a}(\phi)$ denotes $W^{a}\left(\left(\phi^{a}\right)^{-1}(\phi)=t\right)$, where $\left(\phi^{a}\right)^{-1}(\phi)$ is the inverse of the mapping $\phi^{a}(t)$. The analogous notation is used for $W^{b}(\phi)$.
} 


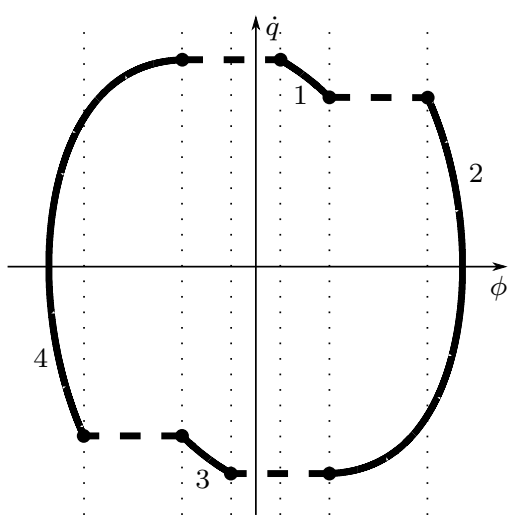

(a) $\epsilon_{\phi}>0,|\hat{\theta}|<2 \epsilon_{\phi}$

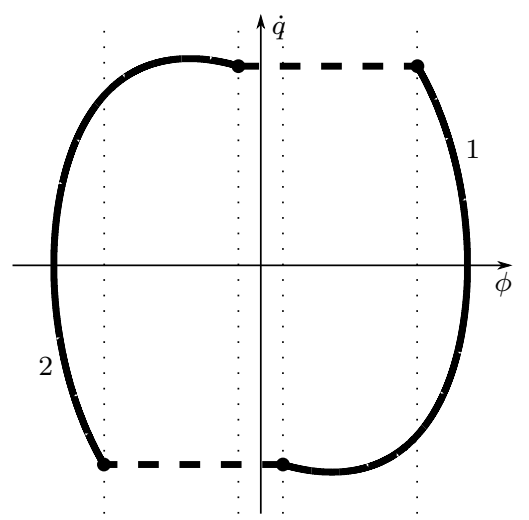

(b) $\epsilon_{\phi}>0,|\hat{\theta}| \geq 2 \epsilon_{\phi}$

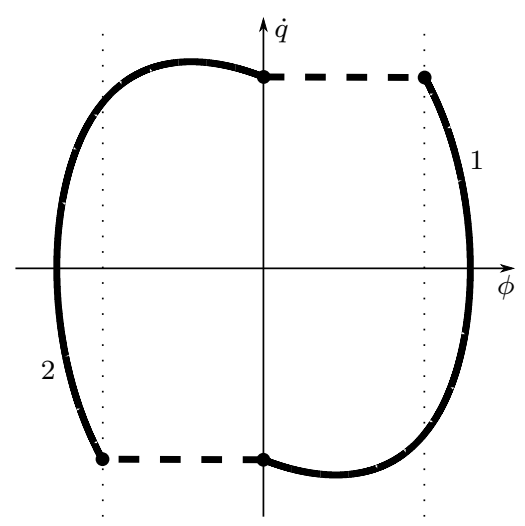

(c) $\epsilon_{\phi}=0,|\hat{\theta}|>0$

Fig. 5. Limit cycles in the state plane obtained by simulation. Three different sets of controller parameters $\left(\epsilon_{\phi}, \hat{\theta}\right)$ are compared. Solid lines represent the continuous motion of each individual subsystem. Dashed lines represent the switching.

(S1) the transition condition is approached, but with zero velocity (1.e. the damping of the system is too high leading to an aperiodic motion);

(S2) the transition condition is not approached (this is the case when the "switch on" position of a subsystem corresponds to a lower level of potential energy than the transition condition for the next subsystem, and the kinetic energy at the "switch on" instant is too low).

Scenario (S1) can be avoided, when the solution of the considered system is not aperiodic. Note that this is the only assumption regarding the parameters of the plant (1).

Remark 6. The results of Theorem 4 can be extended to the class of second-order systems including nonlinear damping forces $h(\phi, \dot{\phi})$ satisfying $\dot{W}(\boldsymbol{x})=-h(\phi, \dot{\phi}) \dot{\phi} \leq 0$, where for $\dot{\phi}=0, h(\phi, \dot{\phi})=0$ and $|h(\phi, \dot{\phi})| \geq d_{\min }|\dot{\phi}|$ is bounded by either a positive constant $d_{\text {min }}$ or a nondecreasing function $d_{\min }(\|\boldsymbol{x}\|)>0$.

The only case where scenario (S2) might occur is $\left(\epsilon_{\phi}, \hat{\theta}\right) \in$ $\mathcal{P}_{1}$. This is as the transition conditions from $j=1 / j=3$ to $j=2 / j=4$ are at a higher level of potential energy than the "switch on" positions (cf. Fig. 1(a) and Fig. 5(a)). For all other controller parameters, global convergence of the limit cycle can be shown.

From Fig. 5, qualitative statements regarding the efficiency of the limit cycles can be deduced:

- $\mathcal{P}_{1}$ : the controller injects and removes energy, while the amount of injected energy is larger than the removed energy;

- $\mathcal{P}_{2}, \mathcal{P}_{3}$ : the controller strictly injects energy.

Assuming that the energy transfer from the actuators to the load mass is associated to substantial losses in both directions (actuator to load and load to actuator), which is of course the case when, for instance, gear boxes are present, the cases $\mathcal{P}_{2}, \mathcal{P}_{3}$ are more efficient than $\mathcal{P}_{3}$. On the limit cycle corresponding to $\mathcal{P}_{2}, \mathcal{P}_{3}$, the injected energy is completely dissipated by the natural damping properties of the plant. This means that the actuators inject exactly the minimum amount of energy required to sustain the limit cycle and no power flow back to the actuator is performed.

\section{CONCLUSION}

A comprehensive stability analysis for a switching based limit cycle controller is derived. The stability statement is based on multiple, physically motivated Lyapunov functions and extends the results of Branicky (1998) for limit cycles. Furthermore, to verify the basic assumption of the main theorem, a novel interpretation of contraction analysis is introduced. The complete analysis is based on physical considerations and therefore provides detailed insights in the principles of this new concept to generate limit cycles.

The control approach applies for general, nonlinear secondorder dynamics with positive definite stiffness, inertia, and damping properties, where the damping is such that aperiodic motions can be excluded. In particular, for the majority of controller parameters, that is for all $\left(\epsilon_{\phi}, \hat{\theta}\right) \in \mathcal{P}_{2} \cup \mathcal{P}_{3}$, global attractiveness of the limit cycle can be guaranteed. A qualitative efficiency analysis revealed that in case of these controller parameters, the energy efficiency of the limit cycle is higher than in case of controller parameters, where only local attractiveness of the limit cycle can be guaranteed (1.e. $\left.\left.\left(\epsilon_{\phi}, \hat{\theta}\right) \in \mathcal{P}_{1}\right)\right)$. From a view point of energy efficiency, this novel control concept of limit cycle generation can be more advantageous than implementing the classical Van der Pol oscillator by control, since the controller performs only positive work at the plant to sustain the limit cycle.

\section{Appendix A. PROOF OF THEOREM 2}

The first part of the proof is conceptually similar to the proofs in Branicky (1998); Lu and Brown (2010).

Throughout the proof let $\mathcal{S}(r)$ and $\mathcal{B}(r)$ represent the Euclidean sphere respectively ball, with radius $r$ and center at the origin.

First, assume that condition (C1) is satisfied in the repetition $p$ when subsystem $j$ is active. From $\Delta V_{j_{(k+1)}+} \leq c$ it follows that $\forall j \in \Sigma$ there exist $\alpha_{j} \leq p|c|$ such that $V_{j}\left(\boldsymbol{x}_{j}(t)\right) \leq \alpha_{j}$ for all $t \in \bigcup_{k=1 \ldots p}\left[t_{j_{k+}}, t_{j_{k^{-}}}\right]$. Then, for any $R_{j}>\alpha_{j}, \exists r_{j}<R_{j}$ such that for all $t \in \bigcup_{k=1 \ldots p}\left[t_{j_{k^{+}}}, t_{j_{k^{-}}}\right]$ 
and $\boldsymbol{x}_{j}(0) \in \mathcal{B}\left(r_{j}\right), V_{j}\left(\boldsymbol{x}_{j}(t)\right)<\min V_{j}\left(\boldsymbol{x}_{j} \in \mathcal{S}\left(R_{j}\right)\right)$. This is as $V_{j}\left(\boldsymbol{x}_{j}(t)\right)$ is continuous in the active region. Since the above result holds for all $j \in \Sigma$ and $k=1 \ldots p$, all trajectories $\boldsymbol{x}_{j}(t)$ starting inside $\mathcal{B}\left(r_{j}\right)$ stay inside $B\left(R_{j}\right)$ as long as the subsystem $j$ is active. The trajectories $\boldsymbol{x}_{j}(t)$ of each individual subsystem $j$ keep bounded in $B\left(R_{j}\right)$.

Note that if we straighten the boundedness condition such that $\Delta V_{j_{(k+1)+}} \leq 0$ we obtain Lyapunov stability. In that case the theorem would be equivalent to (Branicky, 1998, Theorem 2.3). Since we are interested in the existence of limit cycles, we keep the upper bound condition $V_{j_{(k+1)}+} \leq$ $c$ and additionally introduce the option of condition (C2).

Second, assume that condition (C2) is satisfied for $j=1$, 1.e. the sequence $\left\{\Delta V_{1_{(k+1)}}\right\}$ converges to zero as $k \rightarrow \infty$. Since $V_{j}\left(\boldsymbol{x}_{j}(t)\right)$ is non-increasing for $t \in \bigcup_{k}\left[t_{j_{k+}}, t_{j_{k^{-}}}\right]$, 1.e. $\Delta V_{j_{k}} \leq 0$, further since $k \rightarrow \infty$ implies that the fundamental switching sequence $\Sigma$ is repeated, 1.e. $(j+$ 1) $\in \Sigma$ has the value $\min (\Sigma)=1$ if $j=\max (\Sigma)$, and $\Delta V_{j, j+1}=$ const., we may conclude if $\Delta V_{l_{(k+1)+}}=0$ for $l=1$,

$$
\Delta V_{l_{(k+1)+}}=\sum_{j=l}^{l+(n-1)}\left(\Delta V_{j_{k}}+\Delta V_{j, j+1}\right)=0,
$$

$\forall l \in \Sigma$ such that condition (C2) is satisfied $\forall j \in \Sigma$. Therefore, the sequences $\left\{\Delta V_{j_{(k+1)+}}\right\}$ converge to zero which implies that the series $V_{j^{+}}=V_{j_{1+}}+\sum_{k=1}^{\infty} \Delta V_{j_{(k+1)}}=$ $\alpha_{j}$ such that $V_{j}\left(\boldsymbol{x}_{j}(t)\right) \leq \alpha_{j}$ for all $t \in \bigcup_{k}\left[t_{j_{k+}}, t_{j_{k^{-}}}\right]$. Then, for any $R_{j}>\alpha_{j}, \exists r_{j}<R_{j}$ such that for all $t \in$ $\bigcup_{k}\left[t_{j_{k+}}, t_{j_{k^{-}}}\right]$and $\boldsymbol{x}_{j}(0) \in \mathcal{B}\left(r_{j}\right), V_{j}\left(\boldsymbol{x}_{j}(t)\right)<\min V_{j}\left(\boldsymbol{x}_{j} \in\right.$ $\left.\mathcal{S}\left(R_{j}\right)\right)$. This is as $V_{j}\left(\boldsymbol{x}_{j}(t)\right)$ is continuous in the active region. Since the above result holds for all $j \in \Sigma$ and $k$, all trajectories $\boldsymbol{x}_{j}(t)$ starting inside $\mathcal{B}\left(r_{j}\right)$ stay inside $B\left(R_{j}\right)$ as long as the subsystem $j$ is active. The trajectories $\boldsymbol{x}_{j}(t)$ of each individual subsystem $j$ keep bounded in $B\left(R_{j}\right)$.

Finally, assume that in addition to condition (C2), condition (C3) is satisfied. We have already shown that $\forall j \in \Sigma$, $\Delta V_{j^{+}}=0$, which implies $V_{j_{\infty}}=\alpha_{j}$, where $\alpha_{j}$ is constant. First of all, let $\mathcal{S}_{j}:=\left\{\boldsymbol{x}_{j} \in \mathbb{R}^{2} \mid V_{j}\left(\boldsymbol{x}_{j}\right)=\alpha_{j}\right\}$ and let $\boldsymbol{p}_{j}:=\left\{\boldsymbol{x}_{j} \in \mathcal{S}_{j} \mid \phi_{j}=\right.$ const. $\}$ (note that $\boldsymbol{x}_{j}=$ $\left.\left(\phi_{j}, \dot{q}\right)^{T}\right)$. The points $\boldsymbol{p}_{j}$ are well defined, since the hybrid system is autonomous (state dependent switching) and each individual subsystem $\dot{\boldsymbol{x}}_{j}=\boldsymbol{f}_{j}\left(\boldsymbol{x}_{j}\right)$ is asymptotically stable. Thereby, due to the correspondence (7) (between hybrid and continuous states) the points $\boldsymbol{p}_{j}^{\star}:=\chi_{j}\left(\boldsymbol{p}_{j}\right)$ are connected via trajectory segments $\boldsymbol{x}_{j}^{\star}(t):=\boldsymbol{\chi}_{j}\left(\boldsymbol{x}_{j}(t)\right)$ for $t \in\left[t_{\infty^{+}}, t_{j_{\infty^{-}}}\right]$, where the resulting path is closed in the domain of continuous states (denoted by $\star$ ). Now, let $\mathcal{C}$ be the closed curve connecting the points defined by $V_{j_{(k+1)^{+}}}=\alpha_{j}$, let $\mathcal{C}_{1}$ be the set of points defined by $V_{j_{k^{+}}}=$ $\alpha_{j, 1}<\alpha_{j}$, and let $\mathcal{C}_{2}$ be the set of points defined by $V_{j^{+}}=$ $\alpha_{j, 2}>\alpha_{j}$. Recall that $\Delta V_{j_{(k+1)+}}\left(V_{j_{k^{+}}}=\alpha_{j}\right)=0$. Evidently, $\Delta V_{j_{(k+1)^{+}}}\left(V_{j_{k^{+}}}=\alpha_{j, 1}\right)>0$ and $\Delta V_{j_{(k+1)+}}\left(V_{j_{k^{+}}}=\right.$ $\left.\alpha_{j, 1}\right)<0$. All paths crossing $\mathcal{C}_{1}$ point outward $\mathcal{C}_{1}$ and all paths crossing $\mathcal{C}_{2}$ point inward $\mathcal{C}_{2}$. Therefore, we can conclude the existence of a limit cycle in the region defined by $\mathcal{C}_{1}$ and $\mathcal{C}_{2}$ using the Poincare-Bendixson theorem (see, e.g. (Jordan and Smith, 2007, Theorem 11.1)). Addition- ally, since the sequence $\left\{\Delta V_{j_{(k+1)}}\right\}$ converges to zero as $k \rightarrow \infty$, the system approaches the limit cycle.

\section{Appendix B. PROOF OF THEOREM 4}

In a first step, we prove the theorem for $\boldsymbol{x}_{-}^{a / b}=\boldsymbol{x}_{-}^{a / b_{\star}}$ and in a second step we show that this case implicates the proof for general $\boldsymbol{x}_{-}^{a / b}$.

Assume that for $W(\boldsymbol{x})=\frac{1}{2} m \dot{\phi}+U(\phi)$, the system $\dot{\boldsymbol{x}}=$ $\boldsymbol{f}(\boldsymbol{x})$ satisfies $\dot{W}(\boldsymbol{x})=-d \dot{\phi}^{2}$ such that we have

$$
\begin{aligned}
\Delta W^{a} & =-d \int_{t_{+}}^{t_{-}^{a \star}}\left(\dot{\phi}^{a}(t)\right)^{2} \mathrm{~d} t=-d \int_{t_{+}}^{t_{-}^{a}} \frac{\left(\mathrm{d} \phi^{a}(t)\right)^{2}}{(\mathrm{~d} t)^{2}} \mathrm{~d} t \\
& =-d \int_{\phi_{+}}^{\phi_{-}^{\star}} \dot{\phi}^{a}(\phi) \mathrm{d} \phi=W^{a}\left(\phi_{-}^{\star}\right)-W^{a}\left(\phi_{+}\right)
\end{aligned}
$$

and

$$
\begin{aligned}
\Delta W^{b} & =-d \int_{t_{+}}^{t_{-}^{b}}\left(\dot{\phi}^{b}(t)\right)^{2} \mathrm{~d} t=-d \int_{t_{+}}^{t_{-}^{b}} \frac{\left(\mathrm{d} \phi^{b}\right)^{2}}{(\mathrm{~d} t)^{2}} \mathrm{~d} t \\
& =-d \int_{\phi_{+}}^{\phi_{-}^{\star}} \dot{\phi}^{b}(\phi) \mathrm{d} \phi=W^{b}\left(\phi_{-}^{\star}\right)-W^{b}\left(\phi_{+}\right) .
\end{aligned}
$$

Since $\dot{\phi}^{a}(\phi)>\dot{\phi}^{b}(\phi) \geq 0, \forall \phi \in\left[\phi_{+} ; \phi_{-}^{\star}\right]$, it follows that

$$
\begin{aligned}
-d \int_{\phi_{+}}^{\phi_{-}^{\star}} \dot{\phi}^{a}(\phi) \mathrm{d} \phi & <-d \int_{\phi_{+}}^{\phi_{-}^{\star}} \dot{\phi}^{b}(\phi) \mathrm{d} \phi, \\
W^{a}\left(\phi_{-}^{\star}\right)-W^{a}\left(\phi_{+}\right) & <W^{b}\left(\phi_{-}^{\star}\right)-W^{b}\left(\phi_{+}\right), \\
W^{a}\left(\phi_{-}^{\star}\right)-W^{b}\left(\phi_{-}^{\star}\right) & <W^{a}\left(\phi_{+}\right)-W^{b}\left(\phi_{+}\right), \\
\Delta W^{a b}\left(\phi_{-}^{\star}\right) & <\Delta W^{a b}\left(\phi_{+}\right)
\end{aligned}
$$

which completes the proof for $\boldsymbol{x}_{-}^{a / b}=\boldsymbol{x}_{-}^{a / b_{\star}}$.

Now, let us prove the theorem for general $\boldsymbol{x}_{-}^{a / b}$.

Subdividing the trajectory segments in three parts (Fig. 4):

- $\boldsymbol{x}^{a}(t), \forall t \in\left[t_{+} ; t_{-}^{a \prime}\right]$ and $\boldsymbol{x}^{b}(t), \forall t \in\left[t_{+} ; t_{-}^{b \prime}\right]$, where $\phi_{+}=\phi^{a}\left(t_{+}\right)=\phi^{b}\left(t_{+}\right), \max \phi_{-}^{\star}=\phi^{a}\left(t_{-}^{a}{ }^{\prime}\right)=$ $\phi^{b}\left(t_{-}^{b \prime}\right)$, where $\max \phi_{-}^{\star}$ is the maximal $\phi_{-}^{\star}$ that satisfies $\dot{\phi}^{a}(\phi)>\dot{\phi}^{b}(\phi) \geq 0, \forall \phi \in\left[\phi_{+} ; \phi_{-}^{\star}\right]$;

- $\boldsymbol{x}^{a}(t), \forall t \in\left[t_{-}^{a \prime} ; t_{-}^{a}{ }^{\prime \prime}\right]$ and $\boldsymbol{x}^{b}(t), \forall t \in\left[t_{-}^{b \prime} ; t_{-}^{b \prime \prime}\right]$, where $\phi^{a}\left(t_{-}^{a \prime}\right)=\phi^{b}\left(t_{-}^{b \prime}\right)=\phi^{a}\left(t_{-}^{a \prime \prime}\right)=\phi^{b}\left(t_{-}^{b \prime \prime}\right)=\max \phi_{-}^{\star}$ and $t_{-}^{b \prime}=t_{-}^{b \prime \prime}$ by definition of $\max \phi_{-}^{\star}$;

- $\boldsymbol{x}^{a}(t), \forall t \in\left[t_{-}^{a \prime \prime} ; t_{-}^{a}\right]$ and $\boldsymbol{x}^{b}(t), \forall t \in\left[t_{-}^{b \prime \prime} ; t_{-}^{b}\right]$, where $\phi_{-}=\phi^{a}\left(t_{-}^{a}\right)=\phi^{b}\left(t_{-}^{b}\right)$ and $\dot{\phi}^{a}(\phi)<\dot{\phi}^{b}(\phi) \leq 0$, $\forall \phi \in\left[\phi_{-} ; \max \phi_{-}^{\star}\right]$ and $\max \phi_{-}^{\star}>\phi_{-} ;$

yields

$$
\begin{aligned}
\Delta W^{a} & =-d \int_{t_{+}}^{t_{-}^{a}}\left(\dot{\phi}^{a}(t)\right)^{2} \mathrm{~d} t=-d\left[\int_{t_{+}}^{t_{-}^{a}}\left(\dot{\phi}^{a}(t)\right)^{2} \mathrm{~d} t\right. \\
& \left.+\int_{t_{-}^{a}{ }^{\prime}}^{t_{-}^{\prime \prime \prime}}\left(\dot{\phi}^{a}(t)\right)^{2} \mathrm{~d} t+\int_{t_{-}^{a}{ }^{\prime \prime}}^{t_{-}^{a}}\left(\dot{\phi}^{a}(t)\right)^{2} \mathrm{~d} t\right], \\
\Delta W^{b} & =-d \int_{t_{+}}^{t_{-}^{b}}\left(\dot{\phi}^{b}(t)\right)^{2} \mathrm{~d} t=-d\left[\int_{t_{+}}^{t_{-}^{b}}\left(\dot{\phi}^{b}(t)\right)^{2} \mathrm{~d} t\right. \\
& \left.+\int_{t_{-}^{b \prime \prime}}^{t_{-}^{b}}\left(\dot{\phi}^{b}(t)\right)^{2} \mathrm{~d} t\right] .
\end{aligned}
$$


Then, since $\dot{\phi}^{a}(\phi)>\dot{\phi}^{b}(\phi) \geq 0, \forall \phi \in\left[\phi_{+} ; \max \phi_{-}^{\star}\right]$, implies

$$
\begin{gathered}
-d \int_{\phi_{+}}^{\phi_{-}^{\star}} \dot{\phi}^{a}(\phi) \mathrm{d} \phi<-d \int_{\phi_{+}}^{\phi_{-}^{\star}} \dot{\phi}^{b}(\phi) \mathrm{d} \phi \\
\Longrightarrow \quad-d \int_{t_{+}}^{t_{-}^{a \prime}}\left(\dot{\phi}^{a}(t)\right)^{2} \mathrm{~d} t<-d \int_{t_{+}}^{t_{-}^{b}}\left(\dot{\phi}^{b}(t)\right)^{2} \mathrm{~d} t, \\
\dot{\phi}^{a}(\phi)<\dot{\phi}^{b}(\phi) \leq 0, \forall \phi \in\left[\phi_{-} ; \max \phi_{-} \star\right], \text { implies } \\
-d \int_{\max \phi_{-} \star}^{\phi_{-}} \dot{\phi}^{a}(\phi) \mathrm{d} \phi<-d \int_{\max \phi_{-} \star}^{\phi_{-}} \dot{\phi}^{b}(\phi) \mathrm{d} \phi \\
\Longrightarrow \quad-d \int_{t_{-}^{a \prime \prime}}^{t_{-}^{a}}\left(\dot{\phi}^{a}(t)\right)^{2} \mathrm{~d} t<-d \int_{t_{-}^{b \prime}}^{t_{-}^{b}}\left(\dot{\phi}^{b}(t)\right)^{2} \mathrm{~d} t,
\end{gathered}
$$

and $t_{-}^{a \prime}<t_{-}^{a \prime \prime}$, implies

$$
-d \int_{t_{-}^{a},}^{t_{-}^{a \prime \prime}}\left(\dot{\phi}^{a}(t)\right)^{2} \mathrm{~d} t \leq 0,
$$

we conclude with a similar argumentation as around (B.1) that

$$
\Delta W^{a b}\left(\phi_{-}\right)<\Delta W^{a b}\left(\phi_{+}\right) .
$$

This completes the proof for general terminal points $\boldsymbol{x}_{-}^{a / b}$.

\section{REFERENCES}

A. Albu-Schäffer, O. Eiberger, M. Fuchs, M. Grebenstein, S. Haddadin, Ch. Ott, A. Stemmer, T. Wimböck, S . Wolf, Ch. Borst, and G. Hirzinger. Anthropomorphic soft robotics - from torque control to variable intrinsic compliance. In C. Pradalier, R. Siegwart, and G. Hirzinger, editors, Robotics Research, volume 70 of Springer Tracts in Advanced Robotics, pages 185-207. Springer, 2011.

M.S. Branicky. Multiple Lyapunov functions and other analysis tools for switched and hybrid systems. IEEE Transactions on Automatic Control, 43(4):475-482, 1998.

D.J. Braun, M. Howard, and S. Vijayakumar. Exploiting variable stiffness in explosive movement tasks. In Robotics: Science and Systems, 2011.

G. Garofalo, C. Ott, and A. Albu-Schäffer. Orbital stabilization of mechanical systems through semidefinite lyapunov functions. In Proc. American Control Conference, 2013.

M. Grebenstein and P. van der Smagt. Antagonism for a highly anthropomorphic hand-arm system. Advanced Robotics, 22(1):39-55, 2008.
S. Haddadin, F. Huber, and A. Albu-Schäffer. Optimal control for exploiting the natural dynamics of variable stiffness robots. In IEEE Int. Conf. on Robotics and Automation, 2012.

D.W. Jordan and P. Smith. Nonlinear Ordinary Differential Equations: An Introduction for Scientists and Engineers. Oxford Texts in Applied and Engineering Mathematics Series. New York, 2007.

D. Lakatos, G. Garofalo, F. Petit, Ch. Ott, and A. AlbuSchäffer. Modal limit cycle control for variable stiffness actuated robots. In IEEE Int. Conf. on Robotics and Automation, 2013a.

D. Lakatos, M. Görner, F. Petit, A. Dietrich, and A. AlbuSchäffer. A modally adaptive control for multi-contact cyclic motion in compliantly actuated robotic system. In IEEE/RSJ Int. Conf. on Intelligent Robots and Systems, 2013b.

D. Lakatos, F. Petit, and A. Albu-Schäffer. Nonlinear oscillations for cyclic movements in variable impedance actuated robotic arms. In IEEE Int. Conf. on Robotics and Automation, 2013c.

D. Lakatos, G. Garofalo, A. Dietrich, and A. AlbuSchäffer. Jumping control for compliantly actuated multilegged robots. accepted for publication at ICRA, 2014.

W. Lohmiller and J.-J. E. Slotine. On contraction analysis for non-linear systems. Automatica, 34(6):683-696, 1998.

J. Lu and L.J. Brown. A multiple Lyapunov functions approach for stability of switched systems. In America Control Conference, 2010.

J.-J.E. Slotine and W. Li. Applied Nonlinear Control. Prentice-Hall, 1991.

S. Stramigioli and M. Dijk. Energy conservative limit cycle oscillations. In IFAC, 2008.

L. Tavernini. Differential automata and their discrete simulators. Nonlinear Analysis: Theory, Methods \&6 Applications, 11(6):665-683, 1987.

B. Van der Pol. On "relaxation-oscillations". The London, Edinburgh, and Dublin Philosophical Magazine and Journal of Science, 2(11):978-992, 1926.

J. Zhao and D.J. Hill. On stability and $l_{2}$-gain for switched systems. In Proc. IEEE Conf. on Decision and Control, 2005.

J. Zhao and D.J. Hill. Passivity and stability of switched systems: A multiple storage function method. System \& Control Letters, 57:158-164, 2008. 